\title{
The Pathogenesis of Acute on Chronic Hepatitis B Liver Failure
}

\author{
Zhao-chun Chi, Quan-jiang Dong and Chang-xin Geng
}

Acute-on-chronic liver failure is a characteristic clinical liver syndrome, which should be differentiated from acute liver failure, acute decompensated liver cirrhosis and chronic liver failure. The pathogenesis of ACLF is not fully understood yet. Viral factors and immune injury have been reported to be the two major pathogenesis. This paper reviewed the researches on the pathogenesis of acute on chronic hepatitis B liver failure in recent years, to provide theoretical basis for prompt and accurate diagnosis and treatment of this syndrome. This would benefit for the prognosis and raise the survival rate of patients.

Key words: Pathogenesis; Acute on chronic hepatitis B liver failure; Cytokine; Immune injury

A cute on chronic liver failure (ACLF) is a characteristic clinical syndrome with poor prognosis. The manifestations of ACLF are based on severe chronic hepatitis. The vast majority of patients with ACLF always suffered from cirrhosis and deteriorated liver function. Acute liver failure occurring under general condition is not a good phenomenon. At present, the worldwide definition and diagnostic criteria of ACLF is still controversial.

The common feature of ACLF is that it can contribute to not only liver failure but also multiple organ failure, especially renal failure. Although the clinical features of liver failure are similar, prognosis are different. The mortality of the patients with ACLF in 28 days and 3 months are $33.9 \%$ and $51.2 \%$, respectively. The mortality of acute decompensated liver cirrhosis (ADC) in the 28 days and 3 months are $1.9 \%$ and $9.7 \%$, respectively. ${ }^{1}$ ACLF occurring on the background of chronic liver disease often has obvious precipitating factors, such as bleeding, infection, poisoning. Therefore, ACLF should be differential diagnosed with identification of chronic liver failure, acute hepatic failure (AHF) and ADC.

In China, $80 \%$ ACLF were caused by acute exacerbation of chronic hepatitis B (CHB). The pathogenesis is very complex and has not been fully elucidated. There is no systemic review on the two major pathogenesis including virus factors and immune injury and related cytokines reported in the Department of Gastroenterology, Qingdao Municipal Hospital, Affiliated Hospital to Ocean University of China, Qingdao, Shandong Province 266071, China

Correspondence: Zhaochun Chi, Email: c.z.chow@163.com world.

\section{SPECIFIC T CELL FAILURE}

Long-term persistent HBV infection is an important factor leading to ACLF. Immunological factors may be important factors involved in the pathogenesis of ACLF. Antigen-presenting cells especially dendritic cells (DC) can reduce the number and deteriorate the function of $\mathrm{T}$ cells. While stellate cells and sinusoidal endothelial cells can present HBV antigen stimulation and the signal is too weak for $\mathrm{T}$ cells to tolerate. ${ }^{2}$ The number of regulatory $\mathrm{T}$ cell (Treg) increases significantly and is positively associated with HBV DNA load. T regulating cells (Tregs), composition and function of which can be reflected by $\mathrm{T}$ cell receptors (TCR), play an important role in maintaining immunological tolerance to self and foreign antigens. The most prevalent $\mathrm{T}$ cell receptor $\beta$ chain variation (TCRBV) in $\mathrm{CD} 4^{+} \mathrm{CD} 25^{+}$Tregs in ACLF and CHB patients includs TCRBV11, BV13.1, BV18, BV20. TCRBV20 CDR3 (complement, complementarity determining region 3) modified "TGTGHSPLH" and TCRBV11 "VYNEQ" CDR3 for diagnosis of ACLF. The expression of some co-inhibitory molecules increased on the surface of the $\mathrm{T}$ cells, including PD-1 (programmed death-1, or programmed cell death 1), CTLA-4 (cytotoxic T lymphocyte associated antigen- 4 and Tim- 3 of $\mathrm{T}$ cell immunoglobulin domain and mucin 3 ) and so on. These co-inhibitory molecules, such as PD-1 can significantly inhibit the secretion of IFN- $\gamma$, the function of IL-12 and the 
activity and proliferation of NK cells. High expression of CTLA-4 inhibitory molecules can increase HBV specific proapoptotic protein Bim (Bcl-2 interference medium), mainly through the induction of CD127 low expression, resulting in $\mathrm{HBV}$ specific $\mathrm{T}$ cell apoptosis. At the same time, Bim made $\mathrm{T}$ cells secrete a small amount of IFN- $\gamma$, and not disintegrate into memory T cells. ${ }^{4}$ Tim-3 can reduce the cytokine production. ${ }^{5}$ High expression of multiple co-inhibitory molecules is the key cause to deplete the function of HBV specific $\mathrm{T}$ cell. Therefore blocking these co-inhibitory molecules can contribute to significant restoration of HBV specific $T$ cells and removal of the virus.

The study showd that CHB was characterized by functionally impaired virus-specific $\mathrm{CD} 8^{+} \mathrm{T}$-cell responses, especially increased expression of Bim (a proapoptotic protein). In the body of patients with acute on chronic hepatitis B liver failure (ACHBLF), nonspecific $\mathrm{CD} 8^{+} \mathrm{T}$ cells are dysfunctional, the capacity to produce IL-2 and proliferation of $\mathrm{CD} 8^{+} \mathrm{T}$ cells are significantly decreased, but the function of proinflammatory factor including IFN- $\alpha$ and IFN- $\gamma$ is not damaged, which causes nonspecific inflammatory injury.

\section{STELLATE CELLS IN THE PATHOGENESIS OF ACLF}

The experimental data showed that the HSCs (hepatic stellate cells) also had characteristics of liver stem/progenitor cells (HPCs). Liver biopsy with histochemistry analysis showed that the number of activated HSCs and HPCs was higher in ACLF than in acute hepatitis and other chronic liver disease $(P<0.001)$. Meanwhile it was found that there's a significant correlation between the presence of HSCs and HPCs, which points out the HSCs can initiative liver regeneration and pathobiology of ACLF. Rastogi et al gave a report on liver biopsy of 70 cases of ACLF, which showed significant more $\alpha$-SMA activated HSCs in ACLF biopsies than in biopsies of patients with acute hepatitis, chronic hepatitis, and normal LDLT donor biopsies. ${ }^{6}$ So it suggested a significant correlation between HSCs and ACLF.

\section{THE GENOTYPE OF HBV AND ACLF}

In recent years, a correlation between the HBV genotype and ACLF has been found. It was pointed out in that C53T, A1846T and G1896A are the most common ACLF gene mutations. Cross sectional study showed that $61 \%$ of ACLF patients, $11.1 \%$ of chronic hepatitis B (CHB) patients, $31.1 \%$ of liver cirrhosis (LC) patients and $33.3 \%$ of hepatocellular carcinoma (HCC) patients carried T1864. The percentage of patients with ACLF, CHB, LC, HCC carrying A/ G1913 were $42.9 \%, 2.2 \%, 17.8 \%$ and $11.1 \%$, respectively. In addition, they also found the incidence of T1846 was much higher in patients infected with B genotype (57.1\%) than those with $\mathrm{C}$ genotype (30.4\%). The A/G1913 gene in HBeAg negative patients $(28 \%)$ was higher than that in HBeAg positive patients (13.2\%). Multivariate analysis indicated that T1846 and A/G1913 were independent factors of ACLF, whose gene mutations were closely associated with the pathogenesis of ACLF.

HBV genotype and mutations in patients with ACHBLF are located in the basal core promoter $(\mathrm{BCP})$ and precore $(\mathrm{PC})$ regions. Xiao $\mathrm{L}$ et al have done a survey on 322 patients with chronic HBV infection, including 77 cases of ACHBLF, 109 cases of $\mathrm{HCC}$, and 136 cases of CHB. ${ }^{8}$ Among them, there were $61.5 \%$ patients with $\mathrm{B}$ genotypes, and $38.5 \%$ patients with $\mathrm{C}$ genotypes. The percentage of patients with ACHBLF with B genotype was significantly higher than that with $\mathrm{CHB}(92.2 \%$ vs. $60.3 \%, P$ $<0.001)$, whereas the percentage of patients with ACHBLF with $\mathrm{C}$ genotype with $\mathrm{HCC}$ was higher than that with CHB. With B genotype A1762T/G1764A, A1846T and G1896A gene mutations, the incidence of ACLF was higher than CHB and the incidence of liver failure was also high.

\section{TH17 $\left(\mathrm{CD4}^{+}{ }^{+}\right)$CELLS AND IL-17 AND ACLF}

The Th17 cells associated with liver injury are involved in the pathogenesis of hepatitis B. Th17 cells, whose mechanism is still unknown, can induce immune activation and disease aggravation. ${ }^{9}$ In patients with ACHBLF, Th17 cells and serum IL-17 concentration is significantly higher than that with $\mathrm{CHB}$, chronic asymptomatic HBV carriers and normal control group. Meanwhile it is worth mentioning that the increase degree is associated with immune inflammation. Th17 cell in the peripheral blood of patients with ACLF is also associated with immune inflammation. Associated with INR, MELD score were positively related, while the level of IL-17 decreased gradually with improvement. ACHBLF Treg/Th17 ratio may be related to the prognosis of the 
disease and $\mathrm{HBV}$ persistent infection. ${ }^{10}$

\section{LIPOPOLYSACCHARIDE, TOLL LIKE RECEPTOR AND ACLF}

It has proved in animal model that high serum lipopolysaccharide (LPS) and LPS-MD-2 (lipopolysaccharide myeloid differentiation protein-2/ TLR4, Toll like receptor 4) complex activated NF- $\kappa$ B and cytokines can induce hepatocyte necrosis. Patients with ACHBLF has dynamic changes of LPS in different disease stage, and has the highest level in the peak phase. The dynamic changes of LPS were related with the severity of disease and may induce secondary liver injury. ${ }^{11}$

TLR3 plays a key role in innate immunity and identification of viral pathogens. TLR3 A952T and C1234T are two polymorphisms. Rong et al have done a survey including 452 cases of chronic hepatitis B and 462 cases of healthy control group. ${ }^{12}$ The results indicated that subjects carrying C1234T genotype and TT genotype had 1.42-fold and 2.31-fold increased risk of chronic HBV infection compared to those with $\mathrm{CC}$ genotype. Further analysis showd the prevalence of $\mathrm{C} 1234 \mathrm{~T}$ genotype and $\mathrm{T}$ allele in $\mathrm{CHB}$ patients with ACLF was significantly higher than that in patients without ACLF. These results indicate that TLR3 C1234T distribution is a risk factor for development of chronic HBV infection, especially the CHB-related ACLF.

\section{GLUTATHIONE-S-TRANSFERASE AND ACLF}

Glutathione-S-transferase M3 (GSTM3) belongs to GSTs family, which can contribute to oxidative stress-mediating liver damage. The study of GSTM3 gene promoter methylation and oxidative stress in ACHBLF patients showed that GSTM3 promoter methylation rate of ACHBLF is higher than that of CHB ( $30 \%$ vs. $6.7 \%, P<0.05)$. Plasma malondialdehyde (MDA) and GST levels in patients with CHB were significantly increased. Plasma MDA and GST levels were much higer in ACHBLF patients than in CHB patients. At the same time, in ACHBLF patients, MDA, MELD scores and mortality rate were much higher in methylated group than those in unmethylated group. Severity about GSTM3 gene promoter methylation and oxidative stress may lead to liver injury and disease of ACHBLF. ${ }^{13}$
Glutathione-S-transferase P1 (GSTP1) gene promoter methylation is closely associated with the liver injury caused by oxidative stress in ACHBLF. $\mathrm{Li}$ et al found that 11 of 35 patients with ACLF and 3 of 35 patients with stable hepatitis B displayed GSTP1 promoter methylation, and the difference was significant. ${ }^{14}$ The levels of MDA adducts were significantly higher in patients with liver failure compared with those with $\mathrm{CHB}$, and in the patients with liver failure who had promoter methylation the levels were higher than in those who did not. The results suggest that GSTP1 promoter methylation may promote oxidative stress-associated liver damage in ACHBLF, and oxidative stress is associated with the severity of ACHBLF.

\section{COX-2 AND PPARG AND ACLF}

COX-2 (cyclooxygenase-2) and PPARg (peroxisome proliferator activated receptor gamma) express in most nucleus of hepatocytes. In liver cells of patients with ACLF or CHB, especially the former, COX-2 expression increased significantly. PPARg also increased significantly in liver cells of patients with ACHBLF. In liver cells of patients with ACLF, COX-2 expression is correlated with the MELD score and PPARg is associated with the loading of HBV DNA. Clinical data indicated that in liver cells of patients with ACLF, ALT, TBil, CHOL (low density lipoprotein cholesterol), PT, FIB (fibrinogen) and MELD were much higher than those of chronic liver failure, chronic hepatitis B and normal control group. Hepatic COX-2 expression is a marker to reflect the degrees of inflammation and injury of liver tissue, and PPARg expression in liver is due to chronic HBV infection, which may be a protective mechanism against liver injury. ${ }^{15}$

\section{IL-21 AND ACLF}

Immune mechanism induced by ACHBLF has not been known much. IL-21 is a newly discovered cytokine, involved in autoimmune and inflammatory diseases. Its potential role in ACHBLF is still unknown. As determined by flow cytometry bead array, the serum levels of cytokines and IL-21secretion of $\mathrm{CD}^{+} \mathrm{T}$ cells in peripheral blood mononuclear cells (PBMC) were identified by intracellular cytokine staining. The results showed that in the blood of patients with ACHBLF the 
secretion of $\mathrm{CD}^{+} \mathrm{T}$ cells-IL-21 increased, and IL-21 level in the serum was the highest in HB-ACLF and positively associated with high MELD score and mortality. In the recovery stage of ACHBLF, serum IL-21 level decreased, so did CD4 ${ }^{+} \mathrm{T}$ cells.

Recombinant human interleukin-21 (rhIL-21) stimulates the secretion of PBMC- IL-1 $\beta$, IL-10, IFN $\gamma$ and TNF $\alpha$. Therefore, IL-21 is a causative factor in severe liver disease, which increases in ACHBLF, and severe liver disease. ${ }^{16}$

\section{CONCLUSIONS}

Overexpression of HBsAg in the liver can cause liver cell degeneration and necrosis, HBV genotype and gene mutation are involved in the pathogenesis of ACHBLF. Endotoxemia and hepatic microcirculation of liver failure occur in the process of depletion of $\mathrm{T}$ cells and loss of the function of specific $\mathrm{T}$ cell. Regulatory $\mathrm{T}$ cells increase is another pathogenesis of ACHBLF. Virus, immune injury and cytokines are interact and reciprocal causation which links perplexing, but detailed mechanism remains unknown. This needs further study. Thorough understanding the pathogenesis of ACLFV would be benefitial for the prognosis of ACLF.

\section{REFERENCES}

1. Moreau R, Jalan R, Gines P, Pavesi M, Angeli P, Cordoba J, et al. Acute-on-chronic liver failure is a distinct syndrome that develops in patients with acute decompensation of cirrhosis. Gastroenterology 2013; 144:1426-1437.

2. Michel ML, Deng O, Mancini-Bourgine M. Therapeutic vaccines and immune-based therapies for the treatment hepatitis B: perspectives and challenges. J Hepatol 2011; 54: 1286-1296.

3. Yang J, Yi P, Wei L, Xu Z, Chen Y, Tang L, et al. Phenotypes and clinlcal significance of circulating $\mathrm{CD} 4{ }^{+} \mathrm{CD} 25^{+}$regulatory $\mathrm{T}$ cells (Tregs) in patients with acute-on-chronic liver failure (ACLF). J Tansl Med 2012; 10:193.

4. Schurich A, Khanna P, Lopes AR, Han KJ, Peppa D, Micco L, et al. Roleof the coinhibitory recepyor cytotoxic $\mathrm{T}$ lymphocyte antigen-4 on apoptosis-prone $\mathrm{CD}^{+} \mathrm{T}$ in persistent hepatitis $\mathrm{B}$ virus infection. Hepatology 2011; 53:1494-1503.
5. Wu W, Shi Y, Li S, Zhang Y, Liu Y, Wu Y, et al. Blockade of Tim-3 signaling restores the virus-specific $\mathrm{CD}^{+} \mathrm{T}$-cell response in patients with chronic hepatitios B. Eur J Immunol 2012; 42: 1180-1191.

6. Rastogi A, Bihari C, Maiwall R, Ahuja A, Sharma MK, Kumar A, et al. Hepatic stellate cells are involved in the pathogenesis of acute-on-chronic liver failure (ACLF). Virchows Arch 2012; 461: 393-398.

7. Yan T, Li K, Li F, Su H, Mu J, Tong S, et al. T1846 and A/ G1913 are associated with acute-on-chronic liver failure in patients infected with hepatitis B virus genotypes B and C. J Med Virol 2011; 83: 996-1004.

8. Xiao L, Zhou B, Gao H, Ma S, Yang G, Xu M, et al. Hepatitis B virus genotype B with $\mathrm{G}$ 1896A and A1762T/G1764A mutions is associated with hepatitis B related acute-on-chronic liver failure. J Med Virol 2011; 83:1544-1550.

9. Zhang GL, Xie DY, Lin BL, Xie C, Ye YN, Peng L, et al. Imbalance of interleukin-17-producing CD4T cells /requlatory $\mathrm{T}$ cells axis occurs in remission stage of patients with hepatitis B virus-related acute-on-chronic liver failure. J Gastroenterol Hepatol 2013; 28: 513-521.

10. Xue-Song L, Cheng-Zhong L, Ying Z, Mo-Bin W. Changes of Treg and Th17 cells balance in the development of acute and chronic hepatitis B virus infection. BMC Gastroenterol 2012; 12: 43.

11. Pan C, Gu Y, Zhang W, Zheng Y, Peng L, Deng H, et al. Dynamic changes of lipopolysaccharide levels in different phases of acute-on-chronic hepatitis B liver failure. PLoS One 2012; 7: e49460.

12. Rong Y, Song H, You S, Zhu B, Zang H, Zhao Y, et al. Association of Toll-like receptor 3 polymorphisms with chronic hepatitis B and hepatitis B-related acute-on-chronic liver failure. Infammation 2013; 36: 413-418.

13. Qil L, Zou ZQ, Wang LY, Gao S, Fan YC, Long B, et al. Methylation of the glutathione-S-transferase M3 gene promoter is associated with oxidative stress in acute-on-chronic hepatitis B liver failure. Tohoku J Exp Med 2012; 228: 43-51.

14. Li T, Meng QH, Zou ZQ, Fan YC, Long B, Guo YM, et al. Correlation between promoter methylation of glutathioneS-transferase P1 AND oxidative stress in acute-on-chronic hepatitis B liver failure. J Viral Hepat 2011; 18: 226-231.

15. Niu YH, Yi RT, Liu HL, Chen TY, Zhang SL, Zhao YR. Expression of COX-2 and PPARg in the livers of patients with acute on chronic HBV-related liver faiulure and their relationship with clinic parameters. Zhonghua Gan Zang Bing Za Zhi (Chin) 2011; 19: 511-516.

16. Hu X, Ma S, Huang X, Jiang X, Zhu X, Gao H, et al. Interleukin-21 is upregulated in hepatitis B-related acute-onchronic liver failure and associated with severity of liver disease. J Viral Hepat 2011; 18: 458-467. 